\title{
Violating conformal invariance: Two-dimensional clusters grafted to wedges, cones, and branch points of Riemann surfaces
}

\author{
Hsiao-Ping Hsu, Walter Nadler, and Peter Grassberger \\ John-von-Neumann Institute for Computing, Forschungszentrum Jülich, D-52425 Jülich, Germany
}

(Received 21 November 2004; published 24 June 2005)

\begin{abstract}
Lattice animals are one of the few critical models in statistical mechanics violating conformal invariance. We present here simulations of two-dimensional site animals on square and triangular lattices in nontrivial geometries. The simulations are done with the pruned-enriched Rosenbluth method (PERM) algorithm, which gives very precise estimates of the partition sum, yielding precise values for the entropic exponent $\theta\left(Z_{N} \sim \mu^{N} N^{-\theta}\right)$. In particular, we studied animals grafted to the tips of wedges with a wide range of angles $\alpha$, to the tips of cones (wedges with the sides glued together), and to branching points of Riemann surfaces. The latter can either have $k$ sheets and no boundary, generalizing in this way cones to angles $\alpha>360^{\circ}$, or can have boundaries, generalizing wedges. We find conformal invariance behavior, $\theta \sim 1 / \alpha$, only for small angles $(\alpha \ll 2 \pi)$, while $\theta \approx$ const $-\alpha / 2 \pi$ for $\alpha \gg 2 \pi$. These scalings hold both for wedges and cones. A heuristic (nonconformal) argument for the behavior at large $\alpha$ is given, and comparison is made with critical percolation.
\end{abstract}

DOI: 10.1103/PhysRevE.71.065104

PACS number(s): 05.50.+q, 05.10.-a

Lattice animals (or polyominoes, as they are sometimes called in mathematics [1]) are just clusters of connected sites on a regular lattice. Such clusters play an important role in many models of statistical physics, and are considered as the standard model for randomly branched polymers [2]. The difference between the animal model and other cluster models such as percolation is that the clusters appear with nontrivial weights in the latter, while every cluster with the same number $N$ of sites has the same weight in the animal ensemble (this gives, more precisely, site animals; in this Communication we shall not consider other models in the animal universality class, such as bond animals or lattice trees [3]). Moreover, lattice animals are one of the few critical models in statistical mechanics violating conformal invariance $[4,5]$. However, the deeper reasons for this violation as well as the consequences are still poorly understood.

On the one hand, the animal problem is similar to other models of statistical physics in allowing a field-theoretic formulation [2] and in showing anomalous scaling laws in space dimensions $d<d_{c}=8$, where $d_{c}$ is called the upper critical dimension. In particular, the number of animals with precisely $N$ sites attached to a given fixed site (the "partition sum"; notice that we count here shifted animals as different) scales for large $N$ as

$$
Z_{N} \sim \mu^{N} N^{1-\theta},
$$

where $\mu$ is the nonuniversal growth constant and $\theta$ is a critical exponent which is independent of the lattice type, but depends on the global geometry of the lattice. Similarly, the gyration radius scales as $R_{N} \sim N^{\nu}$, where the Flory exponent $\nu$ is universal and also independent of the geometry of the lattice.

On the other hand, the statistics of lattice animals have a number of rather unusual features. In particular, as mentioned above, it is not conformally invariant [5]. Conformal invariance gives rather strong constraints in two dimensions. First of all, it gives restrictions on critical exponents. The fact that the Flory exponent $\nu$ is not exactly known for twodimensional (2D) lattice animals (while it is known exactly for unbranched polymers and most other $2 \mathrm{D}$ models) is a consequence of the lack of conformal invariance.

Secondly, and more closely related to the present work, conformal invariance gives strong constraints on the entropic exponent $\theta$ in nontrivial geometries. The most thoroughly studied of such geometries are wedges and cones. A wedge is a part of the plane bounded by two straight lines which intersect at an angle $\alpha$. A cone is basically a wedge where the two boundaries are glued together. A wedge can be mapped onto a half plane by a conformal map, while a cone can be mapped onto a punctuated plane. This implies that for conformally invariant theories the entropic exponent is linear in $1 / \alpha[4-6]$,

$$
\theta=a+b / \alpha .
$$

For models in which one can study single clusters (such as self-avoiding walks, percolation, or lattice animals) this applies to clusters grafted at the tip of the wedge or cone, respectively. While this equation was checked for selfavoiding walks (linear polymers) [6,7], it was indeed found not to hold for lattice animals $[5,6]$. But the numerical results given in the latter papers were not sufficient to suggest any alternative behavior.

In the present Communication we apply the prunedenriched Rosenbluth method (PERM) strategy which was recently adapted to lattice animals [3]. It is a recursively implemented sequential Monte Carlo method with resampling that starts off by growing percolation clusters, re-weighs them according to the animal ensemble, and applies cloning and pruning to achieve approximate importance sampling. It is the most efficient algorithm for simulating animals and lattice trees known today. In particular, it provides very precise estimates of the partition sum, which then allows one to estimate $\theta$ by means of Eq. (1). In the following, the maximal 


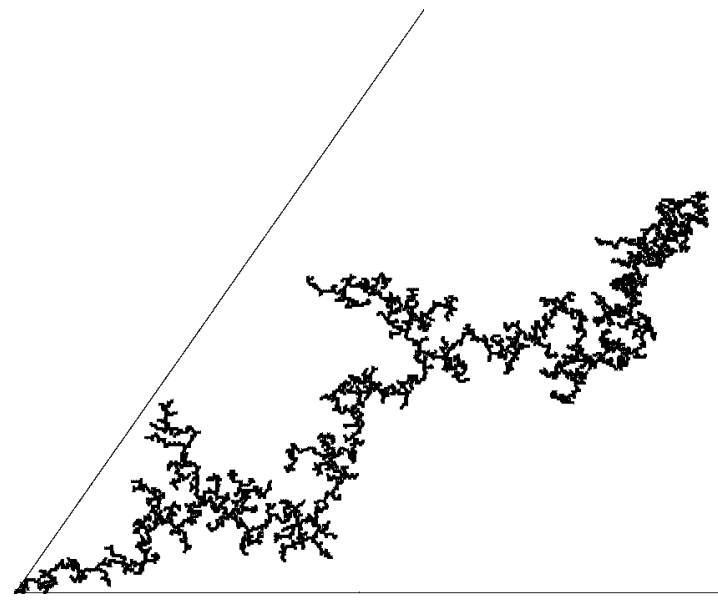

FIG. 1. Typical cluster for a wedge with $\alpha=\pi / 3$ on a triangular lattice; $N=3500$.

sizes of animals varied between $N_{\max }=1000$ and $N_{\max }$ $=4000$.

The results presented below are based on simulations on the square and on the triangular lattices. While the growth constant for the square lattice was taken from [3], we had to perform additional simulations on the full lattice to obtain it for the triangular lattice. We obtained $\ln \mu_{\text {triang }}$ $=1.6454139$ (18) (using the fact that $\theta=1$ on a full $2 \mathrm{D}$ lattice).

Wedges on the square lattice are most easily obtained by placing one edge along one of the coordinate axes (say the positive $x$ axis), and taking the angle $\alpha$ such that $\tan \alpha$ $=n / m$ with $n$ and $m$ being integers. Alternatively, one can place the $x$ axis along the center of the wedge, and use $\tan (\alpha / 2)=n / m$ ("symmetrical wedge"). While these constructions cannot be used, e.g., for wedges of $30^{\circ}, 60^{\circ}$, or $120^{\circ}$, the latter can be obtained for triangular lattices (see Fig. 1). We checked that the standard and the symmetrical wedge gave the same $\theta$ for $90^{\circ}$ (within error bars), and that the square and triangular lattices gave the same results for $180^{\circ}$. Wedges with $360^{\circ}$ are obtained by excluding a single half line.

As we said, cones are obtained by gluing together the two edges of a wedge. This can, of course, only be done if the lattices agree along the two edges and if this does not introduce a line of defects, which strongly restricts the possible angles. For the square lattice, only $\alpha=90^{\circ} 180^{\circ}$, and $270^{\circ}$ are possible, while $60^{\circ}, 120^{\circ}, 240^{\circ}$, and $300^{\circ}$ are also possible for the triangular lattice. Enumeration data for cones with other angles are given in [5], but it is not clear how they were obtained. In any case we checked that defect lines per se have an effect on $\theta$, by simulating animals grafted to points on a defect line.

To simulate angles larger than $2 \pi$, we used multisheeted Riemann surfaces with branch points at the origin where the animal is grafted. A branch point where $k$ sheets meet is essentially a cone with angle $2 k \pi$. Wedges with $\alpha>2 \pi$ are then obtained by cutting out the corresponding domain from the surface.

There are a number of implementation details which have to be specified. For instance, one has to specify for wedges
TABLE I. Entropic critical exponents for 2D lattice animals grafted to the tip of a wedge with angle $\alpha$, and to the branch point (b. p.) of a Riemann surface.

\begin{tabular}{|c|c|c|}
\hline$\alpha$ & $\theta$ & Comment \\
\hline $\arctan (1 / 6)$ & $16.74 \pm 0.05$ & square lattice \\
\hline $\arctan (1 / 5)$ & $14.241 \pm 0.027$ & square lattice \\
\hline $\arctan (1 / 4)$ & $11.741 \pm 0.025$ & square lattice \\
\hline $\arctan (1 / 3)$ & $9.257 \pm 0.016$ & square lattice \\
\hline $\arctan (1 / 2)$ & $6.826 \pm 0.008$ & square lattice \\
\hline$\pi / 6$ & $6.204 \pm 0.008$ & triangular lattice \\
\hline$\pi / 4$ & $4.566 \pm 0.006$ & square lattice \\
\hline$\pi / 3$ & $3.739 \pm 0.006$ & triangular lattice \\
\hline$\pi / 2$ & $2.903 \pm 0.007$ & square lattice \\
\hline $3 \pi / 4$ & $2.316 \pm 0.004$ & square lattice \\
\hline$\pi$ & 2.0 & exact \\
\hline $5 \pi / 4$ & $1.788 \pm 0.006$ & square lattice \\
\hline $3 \pi / 2-\arctan (1 / 2)$ & $1.718 \pm 0.006$ & square lattice \\
\hline $3 \pi / 2$ & $1.622 \pm 0.007$ & square lattice \\
\hline $7 \pi / 4$ & $1.478 \pm 0.007$ & square lattice \\
\hline $2 \pi$ & $1.354 \pm 0.008$ & square lattice \\
\hline $3 \pi$ & $0.790 \pm 0.02$ & square, 2-sheeted b. p. \\
\hline $4 \pi$ & $0.358 \pm 0.02$ & square, 2-sheeted b. p. \\
\hline $6 \pi$ & $-0.660 \pm 0.02$ & square, 3-sheeted b. p. \\
\hline $8 \pi$ & $-1.678 \pm 0.03$ & square, 4-sheeted b. p. \\
\hline $10 \pi$ & $-2.670 \pm 0.05$ & square, 5-sheeted b. p. \\
\hline
\end{tabular}

whether a site on the boundary can be occupied or not. For a cone one might specify whether the two boundaries are identified, or whether they are one lattice unit apart. Finally, for Riemann surfaces the site at the origin can have $\mathcal{N}$ neighbors $(\mathcal{N}$ is the coordination number, $\mathcal{N}=4$ for the square lattice, and $\mathcal{N}=6$ for the triangular one), all of them on one sheet. Or it can have $k \mathcal{N}$ neighbors, occupying all $k$ sheets. We checked in each case several of these alternatives. As expected, they gave different results for finite $N$, but they led to the same scaling behaviors.

Our final results are given in Tables I and II, and are also shown in Fig. 2. Notice that there are two values which are exact: $\theta=1$ for cones with angle $2 \pi$, and $\theta=2$ for wedges with $\alpha=\pi$ [8]. For $\alpha \approx \pi$ our data agree very well with those of [6], but for smaller $\alpha$ the latter data seem to be systematically too low: The value $\theta=5.5 \pm 0.1$ for $\alpha=30^{\circ}$ cited in [6], e.g., is seven standard deviations below our value $6.204 \pm 0.008$. A detailed comparison with the cone data of [5] is less straightforward. For those angles where cones without defect lines exist, the agreement is excellent. For those where there should have been defect lines $\left(127^{\circ}, 143^{\circ}\right.$, $233^{\circ}$ ), the data of [5] seem to be too high by two to four standard deviations (when compared to smooth interpolations of our data), as should be expected from the above discussion.

Both data sets (wedges and cones) confirm that Eq. (2) does not hold. But the wedge data (Table I and Fig. 2) show very clearly that Eq. (2) does hold asymptotically for $\alpha \rightarrow 0$, 
TABLE II. Entropic critical exponents for 2D lattice animals grafted to the tip of a cone with angle $\alpha$, and to the branch point (b. p.) of a Riemann surface.

\begin{tabular}{rcl}
\hline \hline \multicolumn{1}{c}{$\alpha$} & $\theta$ & Comment \\
\hline$\pi / 3$ & $2.5905 \pm 0.0025$ & triangular lattice \\
$\pi / 2$ & $2.1155 \pm 0.0025$ & square lattice \\
$2 \pi / 3$ & $1.8570 \pm 0.0027$ & triangular lattice \\
$\pi$ & $1.5490 \pm 0.0028$ & square lattice \\
$4 \pi / 3$ & $1.3445 \pm 0.0025$ & triangular lattice \\
$3 \pi / 2$ & $1.2549 \pm 0.0028$ & square lattice \\
$2 \pi$ & 1.0 & exact \\
$4 \pi$ & $-0.011 \pm 0.007$ & square, 2-sheeted b. p. \\
$6 \pi$ & $-1.012 \pm 0.009$ & square, 3-sheeted b. p. \\
$8 \pi$ & $-2.020 \pm 0.013$ & square, 4-sheeted b. p. \\
$10 \pi$ & $-3.031 \pm 0.023$ & square, 5-sheeted b. p. \\
$12 \pi$ & $-4.04 \pm 0.04$ & square, 6-sheeted b. p. \\
$14 \pi$ & $-5.045 \pm 0.05$ & square, 7-sheeted b. p. \\
$16 \pi$ & $-6.06 \pm 0.06$ & square, 8-sheeted b. p. \\
$20 \pi$ & $-8.045 \pm 0.07$ & square, 10-sheeted b. p. \\
\hline \hline
\end{tabular}

$\theta(\alpha) \approx 1.35+b_{\text {wedge }} / \alpha \quad$ with $b_{\text {wedge }}=2.543 \pm 0.020$

A similar scaling is also compatible with the cone data, although there the error on $b$ is much larger since we could not go to sufficiently small angles: $b_{\text {cone }}=1.4 \pm 0.1$. On the other hand, both data sets indicate that $\theta$ increases linearly for large angles,

$$
\theta(\alpha) \sim \alpha \text { for } \alpha \rightarrow \infty .
$$

Moreover, the coefficient of proportionality seems to be exactly the same in both cases,

$$
\lim _{\alpha \rightarrow \infty} \frac{\theta(\alpha)}{\alpha}=-\frac{1}{2 \pi}(1.00 \pm 0.03) .
$$

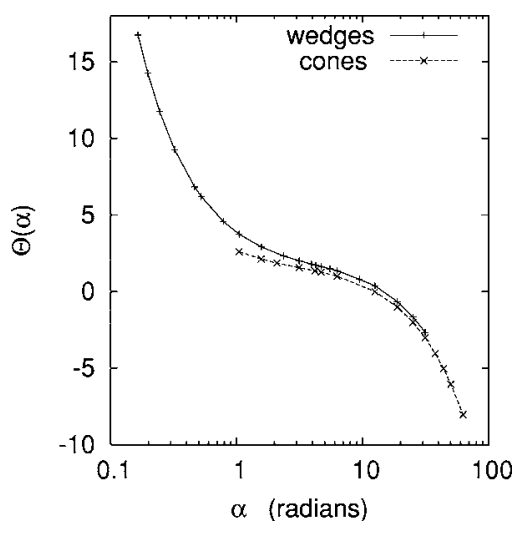

FIG. 2. Values of the entropic exponent $\theta$ for wedges (upper curve) and cones (lower curve) with angle $\alpha$, plotted against $\alpha$. Values for $\alpha>2 \pi$ were obtained using multisheeted Riemann surfaces. Statistical and systematic errors are much smaller than the sizes of the symbols.

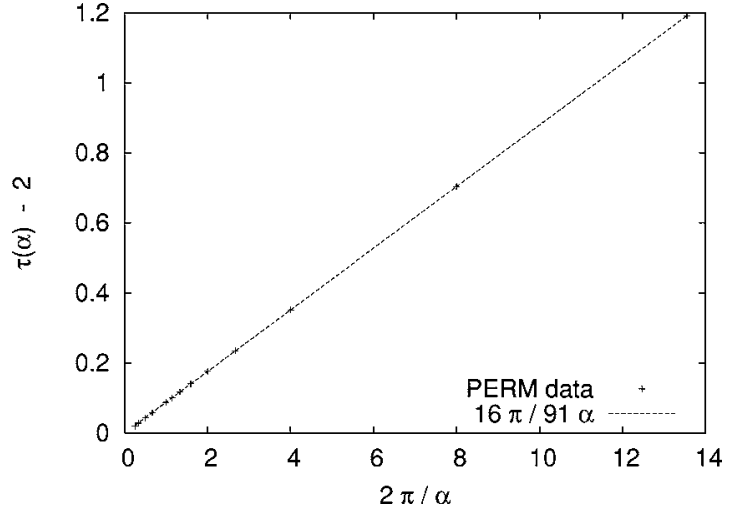

FIG. 3. Values of the exponent $\tau-2$ for critical percolation clusters, grafted on wedges with angle $\alpha$. The points (whose error bars are smaller than the point size) are from simulations for site percolation on the square lattice, the straight line is the theoretical prediction $\tau-2=16 \pi / 91 \alpha$.

In order to understand this behavior heuristically, we now compare lattice animals (which are in the universality class of subcritical percolation) to critical percolation. If we use a standard cluster growth algorithm like the Leath algorithm [9] or the depth-first algorithm of Swendsen and Wang [10] to grow critical or slightly subcritical percolation clusters, the probability of reaching a size $\geqslant N$ decreases like a power [11],

$$
P_{N} \sim N^{2-\tau} f\left(\left(p_{c}-p\right) N^{\sigma}\right) .
$$

Here, $p$ is the wetting probability, $p_{c}$ its critical value, $\tau$ and $\sigma$ are critical exponents, and $f(x)$ decreases exponentially for $x \rightarrow \infty$. The critical exponents can be related to other, more standard, exponents, e.g., [11]

$$
\tau=3-\gamma \sigma \text {. }
$$

The ansatz Eq. (6) holds both for clusters grown in the bulk, and for clusters grown near a surface. In the latter case, $p_{c}$ and the exponent $\sigma=36 / 91$ are the same as in the bulk (and independent of the shape of the surface), while $\tau$ and $f(x)$ do depend on the surface. In particular it is known [4] that $\gamma$ $=25 / 12$ for $2 \mathrm{D}$ clusters attached to a plane wall, giving $\tau(\alpha=\pi)-2=16 / 91$.

From conformal invariance we expect that $\tau(\alpha)$ is a linear function of $1 / \alpha$. On the other hand, we expect that $\tau \rightarrow 2$ for $\alpha \rightarrow \infty$. The reason is very simple: For $\alpha \rightarrow \infty$, the chances that the growth will stop at any finite $N$ will go to zero, since there are ever more possible directions for growth. Thus we expect

$$
\tau(\alpha)=2+\frac{16 \pi}{91 \alpha}
$$

From Fig. 3 we see that this is in excellent agreement with simulations of clusters starting at the tip of a wedge. Notice that the angles shown in Fig. 3 extend up to $8 \pi$, verifying thereby that branch points of Riemann surfaces can be treated like tips of wedges.

For lattice animals one could also try to use Eq. (6), this time with $p \ll p_{c}$, but this would not lead to any useful pre- 
diction. Thus one has to proceed differently. Our main assumption is that clusters will grow essentially into an angular region of size $\Delta \alpha=O(1)$. Much larger angular ranges will also occur, but only with very low probability. For $\alpha \gg \Delta \alpha$, i.e., $k \equiv \alpha / \Delta \alpha \gg 1$, one has thus essentially $k$-independent clusters. If one assumes that all these subclusters have roughly the same size, one expects

$$
Z_{N}(k \alpha) \approx\left[Z_{N / k}(\alpha)\right]^{k} .
$$

Together with Eq. (1) this gives $\theta \propto \alpha$ for large $\alpha$. This argument also explains why the proportionality constant is the same for wedges and cones, but it does not explain its numerical value of $1 / 2 \pi$, i.e., it does not explain why $\theta$ decreases by exactly one unit when $\alpha$ increases by $2 \pi$.

If lattice animals are not conformally invariant but in some way "covariant," one might expect a simple analytical formula for $\theta(\alpha)$. We therefore tried to find such fits. Simple ansatzes like $\theta=a+b \alpha+c / \alpha$ were not successful. The sim- plest acceptable fits were obtained with Padé approximates of the form $\theta=\left(a+b \alpha+c \alpha^{2}+d \alpha^{3}\right) /\left(\alpha+e \alpha^{2}\right)$. But the coefficients $a$ to $e$ looked rather uninspiring, and such an ansatz seems already too complicated for being "natural."

In summary, we have presented very high statistics simulations of clusters grafted to the tips of cones and wedges. For critical percolation clusters these simulations were in full agreement with predictions from conformal invariance. But for lattice animals (subcritical percolation clusters) they agreed with the conformal invariance behavior only in the limit of small angles. For large angles another simple behavior was found and explained by assuming distant angular regions to be essentially independent. Our results clearly show consequences of the violation of conformal invariance in the lattice animals model. Nevertheless, the results also suggest that for small angles conformal invariance may still hold in some approximate way or that some generalized invariance might exist.
[1] S. Golomb, Polyominoes: Puzzles, Patterns, Problems and Packings (Princeton University Press, Princeton, NJ, 1994).

[2] T. C. Lubensky and J. Isaacson, Phys. Rev. Lett. 41, 829 (1978); 42, 410(E) (1979); 20, 2130 (1979).

[3] H.-P. Hsu, W. Nadler, and P. Grassberger, J. Phys. A 38, 775 (2005).

[4] J. Cardy, Nucl. Phys. B 240, 514 (1984).

[5] J. D. Miller and K. De'Bell, J. Phys. I 3, 1717 (1993).
[6] K. De'Bell and T. Lookman, Phys. Lett. 112A, 453 (1985).

[7] A. J. Guttmann and G. M. Torrie, J. Phys. A 17, 3539 (1984).

[8] K De'Bell, and T. Lookman, Rev. Mod. Phys. 65, 87 (1992).

[9] P. Leath, Phys. Rev. B 14, 5046 (1976).

[10] R. H. Swendsen and J.-S. Wang, Physica A 167, 565 (1990).

[11] D. Stauffer and A. Aharony, Introduction to Percolation Theory, 2nd ed. (Taylor and Francis, London, 1992). 